\title{
Energy Efficient Clustering Technique for VANET
}

\author{
Iswarya $\mathrm{B}^{\mathrm{a}, 1}$ and Radha $\mathrm{B}^{\mathrm{b}}$ \\ ${ }^{a}$ Research Scholar in Sree Saraswathi Thyagaraja College, Pollachi, Tamil Nadu, \\ India \\ ${ }^{\mathrm{b}}$ Assistant Professor, Department of Information Technology, Sri Krishna Arts and \\ Science College, Coimbatore, India
}

\begin{abstract}
Vehicular ad-hoc networks (VANET) are listed as an extension of mobile ad-hoc networks (MANETs) which can improve road safety and provide the Intelligent Transportation Systems (ITS). In addition to its advantages, VANET faces many obstacles ranging from high-energy consumption to instability induced by high changes in topology. The main goal of designing an optimum route algorithm is to decrease the probability of contact failure and reduce the energy consumption of nodes within the network. Clustering is thus a method to combine nodes and make the network more robust. With no node consciousness, it is often shorter on resources, which causes network execution problems and changes in topology. At that point, a primary energy issue emerges in the AODV routing protocol that aims to improve the energy efficiency of the $\mathrm{V} 2 \mathrm{~V}$ communication in nodes lifetime and to connection lifetime problems in the network. This article proposed a clustering-based optimization technique called Energy Efficient Clustering Technique (EECT) with the AODV protocol's K-Medoids clustering algorithm in order to cluster vehicle nodes and find nodes that are convincing to interact in a defined secured and reliable path, which detailed in previous works. Efficient nodes are recognized from each cluster with the goal of energy-efficient communication, to optimize the parameter as minimum energy consumption in VANET.
\end{abstract}

Keywords. VANET, AODV, Energy Efficient Routing, K-Medoid Clustering, Minimum Energy Consumption.

\section{Introduction}

Electronic road signs were visible throughout the mid-19th century, traffic officers used colored lights, hand signals, semaphores to track and monitor farmer movements in the 1930s. The 1940s saw widespread use of vehicle indicators, and in the 1960s various types of warning signals were introduced to alert drivers to current events [1]. Countries like the United States, Japan and Europe have recently launched measures to create a safer and more efficient driving situation. It has led researchers to engage in vehicle technology or intelligent transport systems and to exchange vast amounts of information, such as traffic information and direction, through wireless network protocols. This led

\footnotetext{
${ }^{1}$ Iswarya B, Sree Saraswathi Thyagaraja College, Pollachi, Tamil Nadu.

E-mail: iswaryabalu@gmail.com.
} 
to the development of an ad hoc vehicle network called the Vehicular Ad-hoc Network (VANET). VANET is a mobile ad-hoc network that tackles all issues related to the messaging of wireless test vehicles. An ad-hoc network is a set of mobile nodes that usually have wireless connectivity without any current or centralized connection point [2]. VANET allows the creation of wireless vehicles as necessary. Comprehensive path must begin to allow efficient communication of the packet to its destination through reasonable routing protocol architecture. As a result, transceivers and computerized vehicle control modules enables communication [3]. The aim of VANET is to ensure comfort and protection in fuel stations, weather, parking, traffic blocks and emergency alerts. Abundant energy is wasted in the node during transmission and battery life in order to provide this information. The VANET architecture is detailed in Figure 1.

The vehicle data is handled by computers, sensors, automobile foundations and equipment. VANET, no network provider, provides road (vehicle) and roadside (RSU) connectivity to customers [4]. V2I, V2V and VRC [5] have been found in Intelligent Transportation Systems (ITS) areas to resolve some of the different issues.

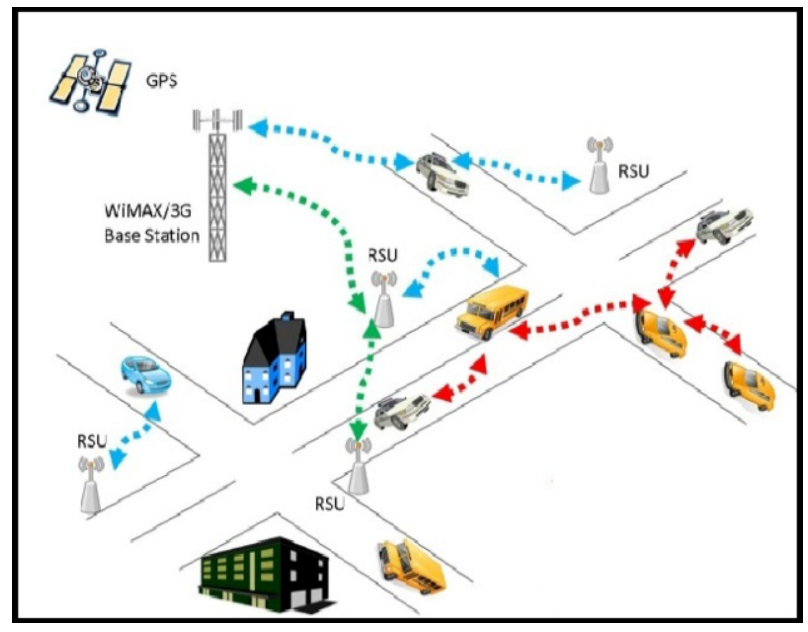

Figure 1. VANET Architecture.

Over the years Green Technology has taken the growing use of electricity in remote technologies into consideration. The complex technical collection includes factor in transmission power and transmission probability. Every round, nodes will send traffic and use their energy; nodes will refresh the chances of sending them on prior conviction. The supplementary protocol improves energy efficiency and reduces dead nodes in the Wireless Network. The algorithm is used in critical physical inactivity and energy efficiency network [3,5,6], which is often missing. The Energy-Efficiency Protocol reduces the WSN's energy consumption and thus expands its operating range, leading to the use of the shortest possible routes. Once, it is attempting to change the use of certain power to predict network clustering. Therefore VANET, incorporates dynamic topology as well as some arbitrary and variable network forecasts for vehicle speed. The best way to move data from the source node to the target node is to analysis to determine the proper moving of vehicles to transmit information related to traffic, between the source node and the destination node. Interestingly, various governments, companies and aca- 
demic institutions around the world have taken over numerous VANET companies in the last decade.

\section{Related Works}

The study was inspired by some work on the VANET energy supply. Here are some of the major inquiries. Lochert et al. [7] suggested that they use a genetic algorithm to approach a traffic information system (RSU). Good RSU sites are chosen from the initially defined list of possible positions to assess the extremely fragmented nature of VANET during the early deployment phase. The choice of the best set of RSUs, however, strongly depends on the simulated transport situation in the studies.

In the road scenario, the study of VANET support location routing (LAR) using metrics such as packet delivery ratio (PDR), network performance, average delay and overhead routing was presented by Husain et al. [8]. The LAR for various node density metrics is calculated. The test was performed at $100 \mathrm{~km} / \mathrm{h}$ at some very high speed. The protocol demonstrated good communication efficiency in vehicle, showing that, at a fair number of nodes, the PDR increases and although decreases the higher network density.

Zhang et al. proposed energy efficient routing Protocol (ERBA) [9] that uses vehicle activity and classification of VANET motion patterns. Changing patterns are introduced, considering current and future trends. ERBA allows packet transmission with a proactive routing protocol to choose appropriate paths for applications that require delays. ERBA analysis and correlation in Shanghai with AODV real urban scenarios, considering such performance metrics as performance levels, probability density, reliable neighborhood connection and end-to-end delay.

Deshmukh and Sonekan [10] proposed an improved AODV protocol to improve the performance of the standard AODV protocol, in order to improve the route discovery process, improve the road detection process and reduce energy utilization during message distribution by a two-tier method. Similarly, the improved protocol used reduces the overhead gap and is adaptable to different traffic conditions.

In order to select the correct route from source to destination for energy transmission from a vehicle, Laroiia and Lekhi [11] implemented of the root and leaf node concept using the Location-Aided Routing (LAR) protocol, which defined root nodes. Data can be flowing from the leaf nodes to the root node, usually to the sink. The simulation of 35 nodes and 50 nodes reveals an increase in energy consumption of $56.7 \%$ and $38 \%$ and a decrease of $64 \%$ in 8 seconds, respectively.

The WSN test is comparatively about the limited node energy resources, as Ghaffari relies heavily on WSNs during their lifetime [12]. Therefore, this study explores the effect on transmission decisions in an effective location-based routing protocol and focuses on estimated data transmission for specific service quality $(\mathrm{QoS})$ parameters. While VANET is considered to be a MANET subclass, Samira Harrabi et al. [13] presented VANET can use the high flexibility of the vehicle which ensures successive changes in network topology, including road change and fluctuating node thickness of existing vehicles along the route.

Sharma et al. [14] looked at the methods of integrating the DCF-MAC protocol with an ad hoc power-efficient network of OFDM radio network interfaces. Agarwal et al. [15] proposed a new load-adjusted routing approach to increase grid resilience and battery 
life at each node. Nearly load-adjusted routing study sets these upper limits on two RSUs in rear-to-back for the variable energy transfer dimensions of any vehicle. The problem of a straight net with a uniform distribution of vehicles was identified with more than 1-D lane.

\section{AODV \& K-MEDOID Clustering}

\subsection{AODV Protocol}

The aim of the AODV protocol was to pick an appropriate route from source to destination. AODV [16] starts route scanning when a source node sends data to a destination node. A route request packet (RREQ) is transmitted to the source node during the course of this method. Neighboring nodes clueless of the active route for the corresponding destination node forwards the packet to their neighbors until they locate the active route or hit the maximum hops. Through Unicast mode route reply (RREP) packet is sends back to the source node where the intermediate node knows the path to the target node. Finally, the RREP packet is given to the source node, and the route opens.

Route Discovery-AODV sends RREQs to nearby nodes to investigate the route. The RREQ broadcast includes source address, location of sequence number, broadcast Id, and counter that count the number of times RREQ has been created for a particular node. By increasing the number of hop nodes to minimize RREQ retrieval, it receives RREP from its neighbors or transmits the RREQ from source. Whereas, the RREQ is also conveyed from neighbours. Using the AODV route tab to prevent node entries that do not exist in the source-to-target direction. Route table monitoring is achieved using aim sequence numbers. When a node detects a non-valid communication path, all relevant entries for these invalid routes are removed from the routing table. This then transfers the RREP to neighbouring nodes, no longer true.

\section{2. $K$-Medoid Clustering}

This cluster model uses information pieces " $k$ " to classify $k$ clusters as the initial medoids. Similar residual nodes are positioned in a closest cluster. Until then the Medoid was resolved and could help characterize a cluster, $\mathrm{K}$ clusters focusing on medoids are built, and each individual is placed in the related cluster focusing on the nearest medoid.

K-Medoids [17] or PAM clustering algorithm that is similar to the K-means algorithm. Algorithms are split between K-means and k-medoids (group breaking data). Both would be attempting to reduce the gap between cluster points and center point of the cluster. In comparison to k-means, k-Medoids use data points as a center (medoids or examples) and can be used for arbitrarily defined distances, whereas k-means do not usually use cluster data center (average cluster). In 1987, regular and other remote functions were introduced with PAM. Initialization: Chooses the $n$ data points value ' $K$ ' as the medoids. Selection of Medoids: Medoid value is determined by measuring distance from each of the two data points for any object considered. Here, the distance measure is calculated by Eq. (1).

$$
D_{d i s t}=\left(\sum_{k=1}^{N}\left|v_{i k}-v_{j k}\right|^{n d}\right)^{\frac{1}{n d}}
$$


When $\mathrm{nd}=1$, it is the L1 norm distance metric, when $\mathrm{nd}=2$ it is L2 norm distance metric,and so on. The L1 norm represents the absolute difference between two vehicle attributes. The standard L1 is flexible, robust, and outliers-resistant. Additionally, due to the inherent sparsity, it is computationally efficient in high-dimensional data. To find the distance between vi and vj a co-occurrence based approach is specified in Eq. (2).

$$
D_{\text {dist }}=\frac{1}{K} \sum_{j=1}^{k} \max _{j \neq 1} \operatorname{dist}_{i, j}
$$

Where $D_{i j}$ is when the vehicle vi and vjwithin the same cluster. When vehicle vi and vjare in a different cluster then the Eq. (3) is processed.

$$
D_{i, j}=\frac{\left(\overline{\operatorname{dist}_{i}}-\overline{d i s t_{j}}\right)}{d i s t_{i j}}
$$

Where $\overline{\text { dist }_{i}}$ is the average distance from each point of the ith cluster to the centroid of the ith cluster. $\overline{d i s t}{ }_{j}$ is the average distance from each point of the $i^{\text {th }}$ cluster to the centroid of the $j^{t h}$ cluster. is the distance between the centroids of the $i^{\text {th }}$ and $j^{\text {th }}$ clusters. The maximum value of represents the worst case between the cluster ratio i. Some of the key points to be noted.

1. The initial cluster is created by assigning the closest medoid value to each individual.

2. In each cluster, the role of calculating the new medoid is to minimize the total distance between objects in the cluster.

3. The clustering effects are accomplished by allocating each client to the shortest medoid. Execution of the clustering algorithm may end if the number is equal to something like the previous one. Additionally, the Medoid cycle rehash the K-medoid algorithm for distance calculation of a K-medoid algorithm gets 200 knots initially.

\subsection{Energy Efficient Clustering technique}

This section proposed a K-medoid algorithm involving initialization, iterative detection, and outer detection. DBI-measuring is the basis of assessment cluster algorithms, and the distribution of points is centred on each medoid-like dataset.

A city map can be viewed as a subset of topology for the network, and road transport conditions are limited to vehicle development. Here 200 nodes were used, ordinary nodes showed without a GPS in grey data trading. Conversely, despite the output impedance of the jammers, a few reference nodes must be fitted at once with accurate GPS and poorly controlled communications to provide fair knowledge of the position data for the entire network. There are two routes to this road. All sides of the road have separate source node and destination node; the proposed K-Medoid algorithm is exhibited in Figure 2.

The findings revealed several methods for VANET clustering. Cluster utilises and uses VANET cluster systems for frame clusters, without taking into account a comparison of VANET size. Outside, experts use a model for the K-Medoid clustering that included clustering 200 vehicle nodes, rejecting a lower-individual group of vehicles, and joining with its head of the neighboring group. 


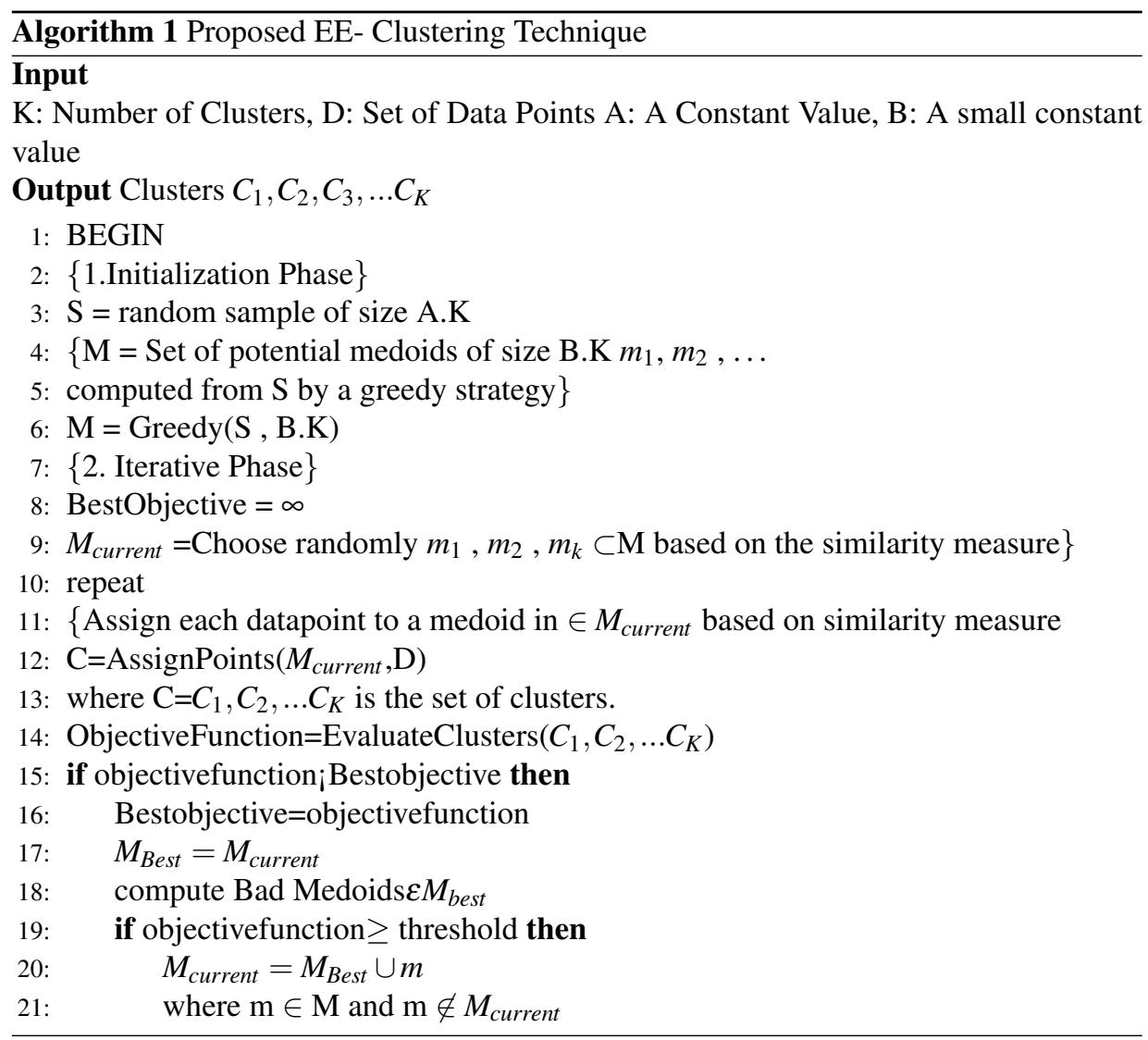

\section{Experimental Evaluation}

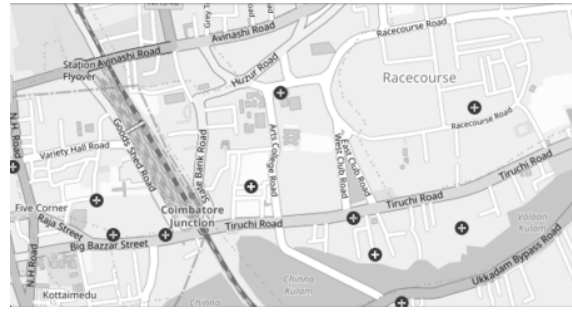

Figure 2. Simulation area

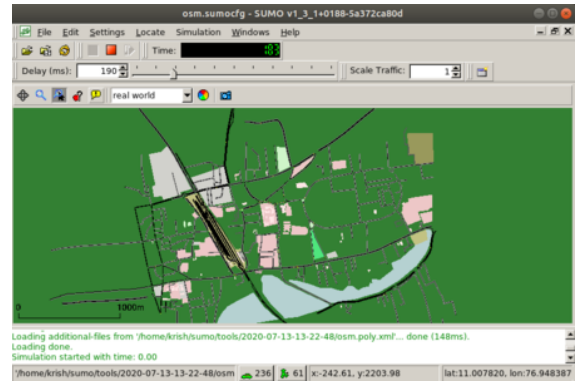

Figure 3. SUMO running environment

The performance of the K-medoid clustering and optimization model is evaluated in terms of energy consumption, packet delivery rate, network throughput, by evaluating the network parameters. To check the efficiency of the study, a Network simulation tool version 3.25 (NS 3.25) is used. With openstreet.org simulation surface was chosen to select the map location and simulated using the SUMO Traffic Simulator. Figure 2 shows 
Table 1. Simulation and traffic specification

\begin{tabular}{|l|l|}
\hline Parameter & Value \\
\hline Protocol & AODV \\
\hline Connection Type & UDP \\
\hline Packet Sizes & 500 Bytes \\
\hline Number of Connections & $10 \%$ of connections \\
\hline CBR rate & $128 \mathrm{~KB} / \mathrm{s}$ \\
\hline Number of simulation run & 10 \\
\hline I-5s length L & $12 \mathrm{Km}$ \\
\hline Number of lanes & 4 \\
\hline Junctions & 20 \\
\hline Maximum number of vehicles & 1250 \\
\hline Mobility traces duration & $400 \mathrm{~s}$ \\
\hline Road length L & $2 \mathrm{Km}$ \\
\hline Traffic lights & 2 \\
\hline Traffic status & continues arrival \\
\hline
\end{tabular}

selected simulation area. SUMO is a microscopic, open-source road traffic simulator, GPL. The German Aerospace Centre (DLR) and ZAIK are launching SUMO (Institute for Applied Informatic Technology). Table 1 lists simulation and traffic detail.

1. Throughput: Network performance can be described as executing accepted packets via a channel.

2. Packet to the Delivery ratio (PDR): The number of packets delivered via communications can be specified as having successfully transmitted a number of packets.

3. Energy Consumption: The overall energy consumption of the packet is called energy consumption from source to destination.

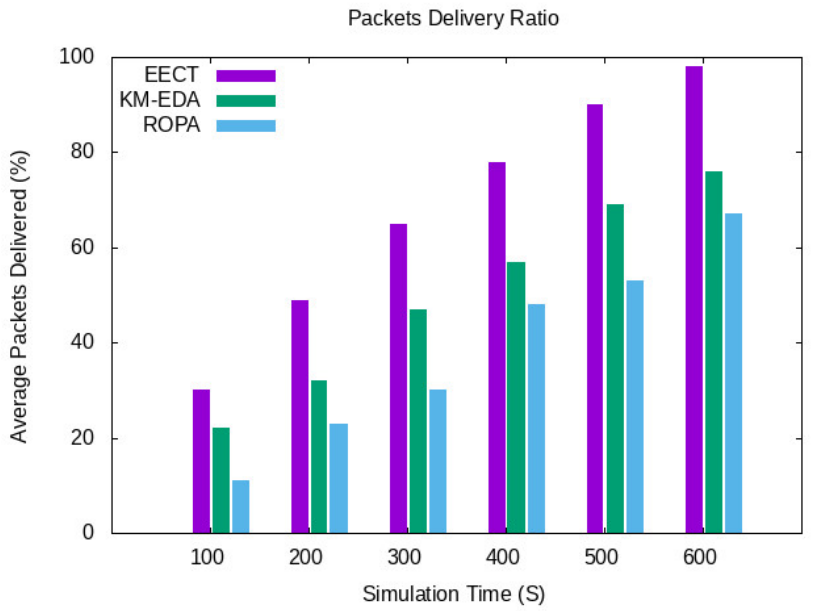

Figure 4. Average PDR 
The running environment of SUMO is presented in Figure 3. Figure 4 presents the PDR between the proposed EECT and existing KM-EDA and ROPA models. EECT maintains better PDR than the existing schemes. It achieves $30 \%$ of better performance in PDR than the others.

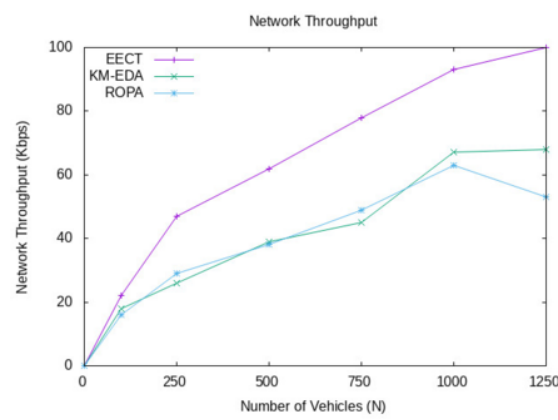

Figure 5. Network Throughput

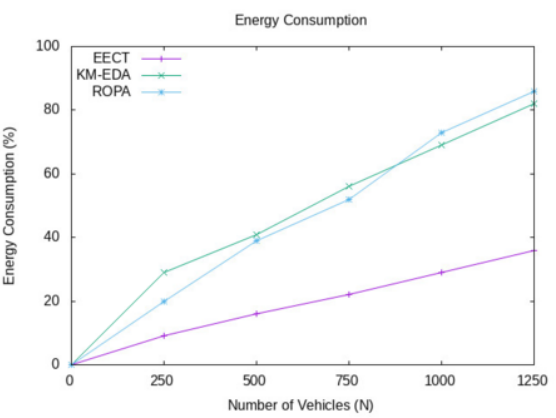

Figure 6. Energy Consumption

Figure 5 shows the network throughput between the EECT and other models. The proposed model attains $40 \%$ or better performance results in throughput compared to the existing. Figure 4 and 5 shows improvements, which in turn results in minimal energy consumption for the proposed model. Therefore, Figure 6 shows the energy consumption model. The proposed EECT retains less energy consumption than the existing systems. The proposed model achieves an energy-saving improvement of $45 \%$ relative to all of the others. Overall, the proposed study achieves an increase of 40 percent in clustering, lifetime and network maintenance compared with the other existing systems. The proposed work thus proved to enhance the VANET strategy for implementing it in Indian road conditions.

\section{Conclusion}

This paper represents VANETs to efficiently classify nodes, taking into consideration as the minimum energy usage. For example, the clustering algorithm, the K-medoid algorithm combined together with optimisation to achieve this goal. It groups the vehicle nodes in different conditions and selects a few other nodes as cluster heads in all other rounds. It will decrease the amount of messages sent from each node to various nodes and base station, saving more energy in the network. By using the EECT algorithm to advance the clustering and the energy-efficient route for V2V communication was obtained. In comparison to the k-medoid approach, the proposed algorithm k-medoid gives the minimum energy consumption resulting from the simulation experiment. This paper concludes the three iteration process that are, i) identifying the stable and secured routing, ii) identifying the reliable path selection for VANET and encompassing it with the stable and secured routing and finally, iii) identifying energy efficient clustering in the above merged work in claiming better model to implement VANET. In future, extend this work by enhancing energy efficiency and optimizing QoS by proposing eventually successful clustering and optimization strategies in Indian regions. 


\section{References}

[1] Tomar R, Prateek M, Sastry GH. Vehicular adhoc network (vanet)-an introduction. International Journal of Control Theory and Applications. 2016;9(18):8883-8.

[2] Omar HA, Zhuang W. Introduction. Springer; 2014 Sep 2.

[3] Hartenstein H, Laberteaux K, editors. VANET: vehicular applications and inter-networking technologies. John Wiley \& Sons; 2009 Nov 4.

[4] Bakshi P, Nandi S. Secure, Privacy Enhanced and Anonymous Communication between Vehicle and Infrastructure. In 2019 Second International Conference on Advanced Computational and Communication Paradigms (ICACCP) 2019 Feb 25 (pp. 1-10). IEEE.

[5] Iswarya B, Radha B. Detailed Survey on VANET Environment. International Journal of Creative Research Thoughts. 2018;6 (1):16-20.

[6] Chaudhary B, Singh S. An Insight Analysis of Economic and Legal Challenges in VANET. International Journal of Applied Engineering Research. 2018;13(17):12991-4.

[7] Lochert C, Scheuermann B, Wewetzer C, Luebke A, Mauve M. Data aggregation and roadside unit placement for a vanet traffic information system. In Proceedings of the fifth ACM international workshop on VehiculAr Inter-NETworking 2008 Sep 15 (pp. 58-65).

[8] Husain A, Kumar B, Doegar A. A study of Location Aided Routing (LAR) protocol for vehicular ad hoc networks in highway scenario. International Journal of Engineering and Information Technology. 2010;2(2):118-24.

[9] Zhang D, Yang Z, Raychoudhury V, Chen Z, Lloret J. An energy-efficient routing protocol using movement trends in vehicular ad hoc networks. The Computer Journal. 2013 Aug 1;56(8):938-46.

[10] Deshmukh P, Sonekar S. Improving Energy and Efficiency in cluster based VANETs through AODV Protocol. IJCSIT) International Journal of Computer Science and Information Technologies. 2014;5(3):4788-92.

[11] Laroiya N, Lekhi S. Energy efficient routing protocols in vanets. Advances in Computational Sciences and Technology. 2017;10(5):1371-90.

[12] Ghaffari A. Hybrid opportunistic and position-based routing protocol in vehicular ad hoc networks. Journal of Ambient Intelligence and Humanized Computing. 2020 Apr;11(4):1593-603.

[13] Harrabi S, Jaafar IB, Ghedira K. Message dissemination in vehicular networks on the basis of agent technology. Wireless Personal Communications. 2017 Oct;96(4):6129-46.

[14] Sharma R, Malhotra J. Performance Evaluation of AODV and GOD for Qos Aware Applications through Realistic Conditions in VANET. International Journal of Computer Network and Information Security. 2015;7(11):64-71.

[15] Agarwal Y, Jain K, Karabasoglu O. Smart vehicle monitoring and assistance using cloud computing in vehicular Ad Hoc networks. International Journal of Transportation Science and Technology. 2018 Mar 1;7(1):60-73.

[16] Kumar A, Sinha M. Design and analysis of an improved AODV protocol for black hole and flooding attack in vehicular ad-hoc network (VANET). Journal of Discrete Mathematical Sciences and Cryptography. 2019 May 19;22(4):453-63.

[17] Elhoseny M, Shankar K. Energy efficient optimal routing for communication in VANETs via clustering model. In Emerging Technologies for Connected Internet of Vehicles and Intelligent Transportation System Networks 2020 (pp. 1-14). Springer, Cham. 